This is an Open Access article, distributed under the terms of the Creative Commons Attribution licence (http://creativecommons.org/licenses/by/4.0/), which permits unrestricted re-use, distribution, and reproduction in any medium, provided the original work is properly cited.

doi:10.1017/jfm.2019.771

\title{
Spreading and oscillation dynamics of drop impacting liquid film
}

\author{
Xiaoyu Tang ${ }^{1}$, Abhishek Saha ${ }^{1,2, \dagger}$, Chao Sun ${ }^{3}$ and Chung K. Law ${ }^{1, \dagger}$ \\ ${ }^{1}$ Department of Mechanical and Aerospace Engineering, Princeton University, Princeton, NJ 08544, USA \\ ${ }^{2}$ Department of Mechanical and Aerospace Engineering, University of California San Diego, La Jolla, \\ CA 92093, USA \\ ${ }^{3}$ Center for Combustion Energy, Tsinghua University, Beijing 100084, China
}

(Received 4 April 2019; revised 26 July 2019; accepted 15 September 2019; first published online 25 October 2019)

We herein report an experimental study to explore the effects of impact inertia, film thickness and viscosity on the dynamics of shape deformation of a drop impacting a liquid film. We have identified that the spreading dynamics shows a weak dependence on impact inertia, but strongly depends on the film thickness. For a thick film, the liquid surface deforms and absorbs part of the impact energy, and hence inhibits spreading of the drop. For a thin film, the drop motion is restricted by the bottom solid substrate, promoting spreading. The periodicity of the capillary controlled shape oscillation, on the other hand, is found to be independent of impact inertia and film thickness. The damping of the shape oscillation shows strong dependence on the film thickness, in that the oscillation decays faster for smaller film thicknesses, due to the enhanced viscous loss.

Key words: drops and bubbles

\section{Introduction}

Drop impact on surfaces is critical in many technological and environmental systems, in which the impact is often associated with heat and mass transfer between the drop and the impacted surface. Since the interfacial transport of heat and mass can significantly depend on the contact area, the post-impact evolution of the drop shape has been of substantial research interest over decades (Roisman, Rioboo \& Tropea 2002; Clanet et al. 2004; Yarin 2006; Son \& Kim 2009; Eggers et al. 2010; Tran et al. 2011; Josserand \& Thoroddsen 2016; Wildeman et al. 2016). These large volume of previous work, most of which were performed for drop impact on solid surfaces, has shown that the drop dynamics after impact can be broadly divided into two stages: the spreading stage, during which the drop impacts and spreads, converting kinetic energy to surface energy with the attendant viscous dissipation, until it reaches the state of maximum deformation; and the rebounding stage, during which the drop

$\dagger$ Email addresses for correspondence: asaha@eng.ucsd.edu, cklaw@ princeton.edu 
retracts, rebounds and oscillates, converting between surface and kinetic energies, while dissipating the total energy through viscous loss. The evolution dynamics of the drop shape during the impact process is mainly characterized by the spreading of the drop and the subsequent oscillation behaviour. The drop deformation during spreading and shape oscillation modulates various aspects including the heat and mass transfer efficiency, accumulation of the liquid film and secondary breakup leading to production of satellite droplets.

The spreading behaviour of a drop impacting on and merging with a solid surface has been studied extensively in the literature (Davidson 2002; Roisman et al. 2002; Clanet et al. 2004; Yarin 2006; Son \& Kim 2009; Vadillo et al. 2009; Eggers et al. 2010; Tran et al. 2011; Josserand \& Thoroddsen 2016; Wildeman et al. 2016). The spreading is generally characterized by the parameter known as the 'spreading factor', defined as the maximum spreading drop radius, $R_{\max }$, normalized by the initial drop radius $R: \beta=R_{\max } / R$. A significant number of studies have focused on developing a universal scaling of the spreading factor as a function of the relevant non-dimensional parameters, in order to capture the relative importance of the inertial, capillary and viscous effects at different impact conditions. In the viscous regime, in which kinetic energy is lost mostly through viscous dissipation, the spreading factor depends on both the Weber number $\left(W e=2 \rho U^{2} R / \sigma\right)$ and the Reynolds number $(R e=2 U R / v)$, with $\beta \sim R e^{1 / 5} f\left(W e R e^{-2 / 5}\right.$ ) (Eggers et al. 2010). On the other hand, in the inertial regime, in which the kinetic energy is mostly converted to surface energy, the spreading factor is found to be independent of viscosity, $\beta \sim W e^{1 / 4}$ (Clanet et al. 2004). Here, $U$ is the impact velocity and $\rho, \sigma, v$ are respectively the density, surface tension and kinematic viscosity of the liquid. There are other factors, such as the wettability and roughness of the impacted solid surface, which may affect the spreading behaviour substantially and consequently are included in more detailed scaling for the spreading factor. Those are summarized by Yarin (2006), Tran et al. (2011) and Josserand \& Thoroddsen (2016).

Although the spreading dynamics for drops impacted on solid surfaces is well studied, it is fundamentally different from impacts on liquid surfaces. When a drop impacts on a solid surface, the latter serves as a stagnation plane that limits the drop motion, resulting in spreading of the deformed drop along the solid surface and consequently conversion of the impact kinetic energy into surface energy and dissipation. On the other hand, when a drop impacts on a liquid surface, the liquid surface can also deform and adapt to the impact. The resistant force from the impacted liquid surface is weaker and thus results in reduced spreading, compared to that of impacts on solid surfaces. Furthermore, since the deformation of the liquid surface takes the shape of a crater, the extent of the drop spreading is also limited. As a consequence of these cumulative effects, the spreading factor is expected to be smaller for impacts on liquid surfaces and different spreading dynamics is expected. Despite its striking difference from impact on a solid surface, the spreading dynamics for drop impact on a liquid film is relatively less explored. A number of studies on drop impact on a liquid film (Cossali, Coghe \& Marengo 1997; Rieber \& Frohn 1999; Davidson 2002; Josserand \& Zaleski 2003; Rioboo et al. 2003; Roisman, van Hinsberg \& Tropea 2008; Lagubeau et al. 2010; Gao \& Li 2015; Geppert et al. 2017; Marcotte et al. 2019; Saha et al. 2019) focused on high Weber number impacts, which cause the drop to merge with the film, and subsequently the large deformation of the combined surface leads to crown formation and splashing. However, the spreading dynamics of the drop on the liquid surface can only be observed for an impact that does not result in immediate merging, and the drop maintains its separate existence 
(a)

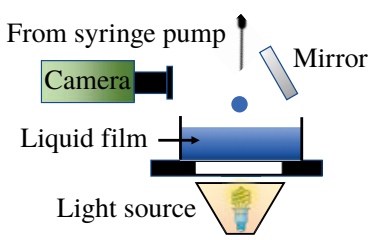

(b)

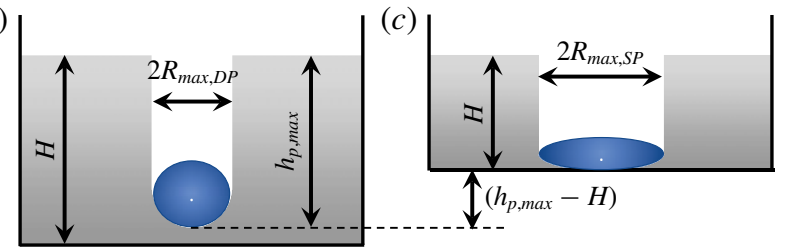

FIGURE 1. (Colour online) Schematic of $(a)$ the experimental set-up, the impact process for $(b)$ a deep pool and $(c)$ a shallow pool.

during the spreading process. This non-coalescing impact can be achieved for an impact with low We, which fails to drain the interfacial gas layer that separates the drop from the liquid surface, subsequently causing the drop to bounce (Pan \& Law 2007; Tang et al. 2016). Furthermore, the low We impacts are widely used for ink-jet printing and 3D printing (Schiaffino \& Sonin 1997), and known for characteristically different drop deformation owing to a stronger effect of surface tension (Wildeman et al. 2016). Thus, in this work, we focus on the spreading of the drop before bouncing from a liquid surface at low We.

The dynamics of shape oscillation of a drop upon impact has been studied in the general context of its primary mode, corresponding frequency and viscous damping (Reid 1960; Miller \& Scriven 1968; Prosperetti 1980; Trinh \& Wang 1982; Tsamopoulos \& Brown 1983; Lundgren \& Mansour 1988; Basaran, Scott \& Byers 1989; Basaran 1992; Becker, Hiller \& Kowalewski 1991, 1994; Schiaffino \& Sonin 1997; Mashayek \& Ashgriz 1998; Trinh, Thiessen \& Holt 1998; van Dam \& Le Clerc 2004). However, most of these studies have focused on freely oscillating drops, such that the effects of large initial deformations due to external disturbances are less explored (Miller \& Scriven 1968; Trinh \& Wang 1982; Basaran et al. 1989; Schiaffino \& Sonin 1997; Trinh et al. 1998; van Dam \& Le Clerc 2004). Furthermore, for impacts on liquid surfaces, oscillation of the drop during the rebounding process is constrained by the presence of the liquid surface, which itself can also deform and oscillate. Characteristics of such oscillating drops have not been adequately explored.

In light of the state of understanding of the subject phenomena, we present herein an experimental and mechanistic study to characterize the dynamics of spreading and oscillation of drops impacting on liquid surfaces. We have quantified the spreading factor, and the amplitude, time scale and viscous decay of the shape oscillation for drops impacting liquid surfaces. We have categorically identified the effects of the impact speed and film thickness on these variables for various liquids covering a large range of viscosities.

\section{Experimental set-up}

In our experiment, the drop was generated by pushing the liquid through a vertically mounted needle at a low speed using a syringe pump. When the drop attains a critical size, it detaches from the needle and falls on the liquid film, which rests in a cubic glass chamber with a cross-section of $25 \mathrm{~mm} \times 25 \mathrm{~mm}$ and a height of $10 \mathrm{~mm}$. The thickness of the liquid film was modulated by adding or removing liquid from the chamber, while the impact speed was modulated by changing the vertical distance between the needle tip and the film. The needle used for these experiments has a diameter of $0.25 \mathrm{~mm}$, which generates drops roughly $1.6 \mathrm{~mm}$ in diameter. Recognizing that the dynamics of spreading and oscillation of the drop is 
Liquids

$\rho$, Density $\left(\mathrm{kg} \mathrm{m}^{-3}\right)$

$\sigma$, Surface tension $\left(\mathrm{mN} \mathrm{m}^{-1}\right)$

$v$, Kinematic viscosity $(\mathrm{cSt})$
n-Tetradecane Silicone oil Silicone oil Silicone oil Silicone oil

$\begin{array}{lllll}\text { C14 } & \text { S1.5 } & \text { S05 } & \text { S20 } & \text { S100 }\end{array}$

763

853

18

913

950

966

$20 \quad 20$

20
20

100

TABLE 1. Properties for the liquids tested.

best characterized in the horizontal plane, the top view of the impact process was accessed through a mirror inclined at $33^{\circ}$ to the vertical axis (shown in figure $1 a$ ). The reflected view of the impact process was then captured at 15037 frames per second (fps) by a high-speed camera (Phantom V7.3, Vision Research) connected to a $50 \mathrm{~mm}$ Nikon lens, a $2 \times$ tele-convertor and a $120 \mathrm{~mm}$ extension bellow to achieve the required image resolution. The optical system was carefully calibrated to account for the distortion created by the inclination. The liquid film was illuminated from the bottom through a diffuser using an Olympus ILP-2 light source. The impact velocity and instantaneous drop radius during the impact were measured from the high-speed images. The liquid film thickness was measured from side view images from a separate camera. Liquids with a wide range of viscosities, listed in table 1, were used in this study.

\section{Global dynamics}

A drop impacting a liquid film can result in two global outcomes, namely, merging, in which the gas layer trapped between the drop and the film interfaces breaks down, causing the drop to merge with the liquid film; and bouncing, in which the gas layer remains intact throughout the impact process, and hence separates the drop from the liquid film. The transition from bouncing to merging outcomes in a parameter space has been examined and scaled for a large number of liquids in our earlier works (Tang et al. 2018, 2019). Recognizing that the spreading and shape oscillation of the drop can only be studied for impacts in which the drop remains separated from the film, this study focuses on the We range where bouncing occurs. Furthermore, it is also noted that, for a given impact velocity (or $W e$ ), if the normalized film thickness, $H^{*}$, is smaller than a critical value, $h_{p, \max }^{*}$, the impact process is affected by the solid substrate at the bottom of the liquid film (Tang et al. 2018). Here, $h_{p, \max }^{*}$ is defined as the normalized maximum penetration depth a drop can attain at a given We when impacting a pool with a large thickness (figure $1 b$ ). The impact condition can thus be broadly divided into two limits: deep pool $\left(H^{*}>h_{p, \max }^{*}\right)$, where the impact is free from the effects of the bottom substrate (figure $1 b)$; and shallow pool $\left(H^{*}<h_{p, \max }^{*}\right.$ ), where the impact is strongly affected by the bottom substrate (figure $1 c$ ). In this study, we have analysed the spreading behaviour and the shape oscillation of the impacted drop for both the shallow pool and deep pool limits.

The spreading and oscillation dynamics has been identified through a sequence of high-speed top view images. A typical impact event is shown in figure 2(a), in which the snapshots are selected at irregular time intervals to reflect the key events during the drop deformation process. A few morphological characteristics can be observed from these images. As the drop impacts and subsequently spreads over the liquid film, surface waves are generated on both the drop and the liquid film surfaces. The surface wave on the liquid film propagates radially outward and does not reflect back 
(a)

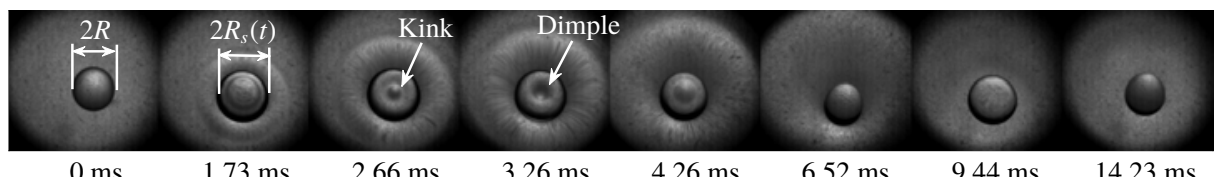

(b)

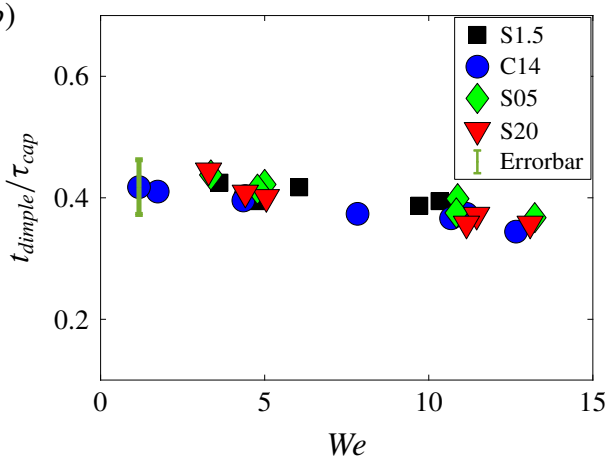

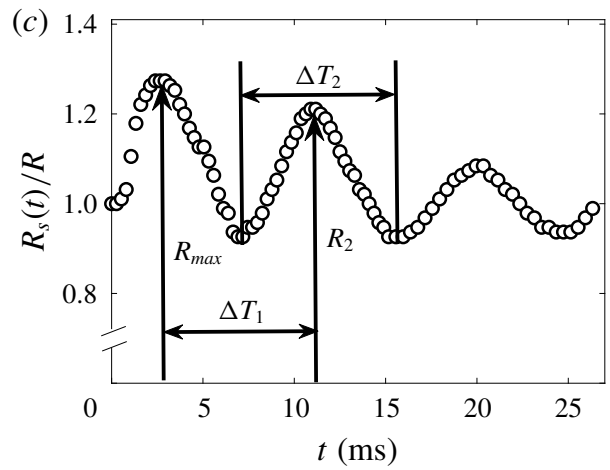

FIgURE 2. (Colour online) Dynamics of drop impact on a liquid film observed from top view. (a) Top view snapshots $\left(\mathrm{C} 14, W e=11.29, H^{*}=3.37\right)$. The initial drop radius $R$ and instantaneous radius $R_{S}(t)$ are measured. (b) Dimple formation time normalized by capillary time scale for all liquids. $(c)$ Time evolution of $R_{s}(t) / R$ measured from top view images. The important time scales and magnitudes are tracked for further analysis.

from the chamber wall, within the duration of experimental observation. On the other hand, the peripheral wave originated at the bottom half of the drop surface propagates longitudinally towards the top half and subsequently degenerates to a point at the north pole, creating features of kink and dimple (figure $2 a$ ). The time taken for the wave to propagate to the north pole to create the dimple, $t_{\text {dimple }}$, is approximately $3.26 \mathrm{~ms}$ for $\mathrm{C} 14$ and is almost constant for all the impact conditions studied (figure $2 b$ ). This time scale is approximately 0.4 of the period for free drop oscillation, i.e. $t_{\text {dimple }} / \tau_{\text {cap }} \approx 0.4$, where $\tau_{c a p}$ is the capillary time scale $\tau_{\text {cap }}=2 \pi \sqrt{\left(\rho R^{3}\right) /(8 \sigma)} \approx 8.43 \mathrm{~ms}$. Ideally, we expect this ratio to be 0.5 assuming that the waves are initiated at the south pole of the drop, and propagate half of the angular wavelength, i.e. $180^{\circ}$, to create the dimple at the north pole. However, in reality, the initial disturbance that creates the surface wave takes place across a finite area around the south pole, and as such reduces the angular distance travelled $\left(<180^{\circ}\right)$, thus the travel time for the wave to reach the north pole. Nevertheless, a near constant $t_{\text {dimple }} / \tau_{\text {cap }}$ across various impact speeds indicates that the formation of the dimple is a characteristic of the drop capillarity. Furthermore, agreements among different liquids show that viscosity also has a weak influence in determining the time scale for the dimple formation (figure $2 b$ ).

The high-speed images shown in figure $2(a)$ were further analysed to characterize the drop shape deformation, by tracking the instantaneous normalized horizontal radius of the drop as it went through different stages of the impact process (figure $2 c$ ). At the spreading stage, the drop spreads over the liquid film until it reaches a maximum spreading radius $R_{\max }$. At the rebounding stage, the drop undergoes about three cycles of oscillations before it completely leaves the liquid surface. Next, we analyse the behaviour of these two stages for all liquids tested and hence identify the effects of $W e, H^{*}$ and viscosity. The important time periods and amplitudes are marked in figure 2(c), which will be discussed in subsequent analyses. 

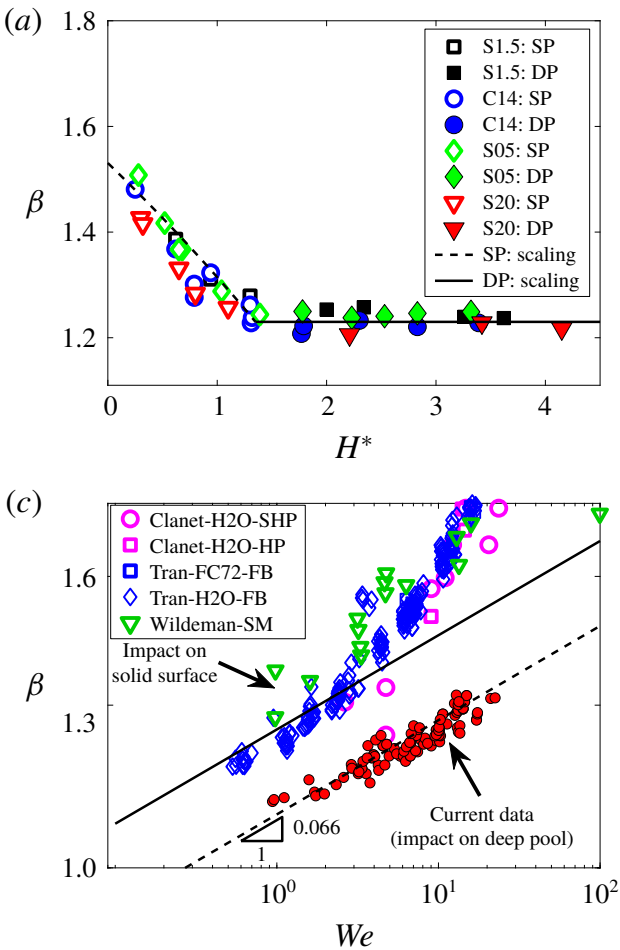
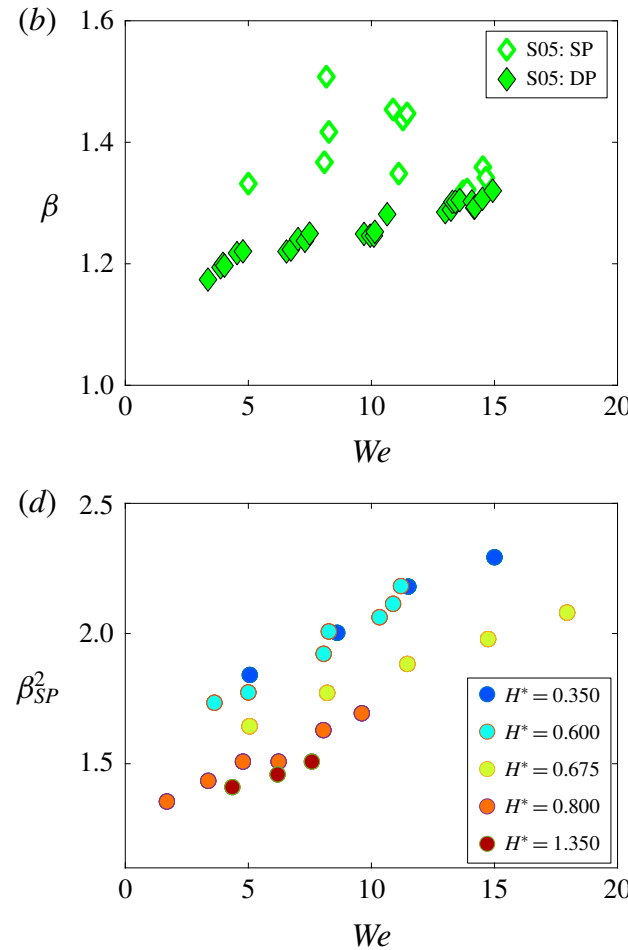

FIgURE 3. (Colour online) Spreading factor $(\beta)$ as a function of $H^{*}$ and We. (a) Value of $\beta$ as a function of $H^{*}$ for all liquids with $8.5<W e<9.75$. SP: shallow pool. DP: deep pool. Scaling is given by (4.2) with $\beta_{D P}=1.23$ and $h_{p, \max }^{*}=1.5$. (b) Value of $\beta$ as a function of We for S05. (c) Value of $\beta$ as a function of We in the deep pool regime for all liquids tested. Red filled circle: current data. Dashed and solid lines: scaling of $\beta \sim W e^{0.066}$. All the other symbols: impact on solid surface adapted from Clanet et al. (2004), Tran et al. (2012) and Wildeman et al. (2016). Clanet-H2O-SHP: experiments with water drop impacting super-hydrophobic surface (Clanet et al. 2004); Clanet-H2O-HP: experiments with water drop impacting hydrophilic surface (Clanet et al. 2004); Tran-FC72-FB: experiments with FC72 drop impacting a super-heated surface inducing film boiling (Tran et al. 2012); Tran-H2O-FB: experiments with water drop impacting a super-heated surface inducing film boiling (Tran et al. 2012). Wildeman-SM: simulation of drop impact on surfaces using slip and no-slip conditions (Wildeman et al. 2016). (d) Value of $\beta_{S P}^{2}$ as a function of $W e$ at different $H^{*}$ for all liquids tested.

\section{Spreading dynamics}

\subsection{Experimental observation}

First, we discuss the experimental results for the spreading factor, $\beta=R_{\max } / R$, measured with a range of $H^{*}$ and We. Figure 3(a) shows $\beta$ as a function of $H^{*}$ for similar values of $W e, 8.5<W e<9.75$, for all liquids. The data from different liquids are represented by different colours and symbols, while the shallow and deep pool limits are distinguished by the open and filled symbols, respectively. It is seen that in the shallow pool limit, the spreading factor decreases with $H^{*}$ and asymptotically attains a constant value at the deep pool limit. As shown in the schematic in figure $1(b)$, for the deep pool, i.e. $H^{*}>h_{p, \max }^{*}$, the drop and liquid 
interface remain far from the bottom substrate such that the drop deformation is independent of $H^{*}$. On the other hand, for the same $W e$ in the shallow pool, i.e. $H^{*}<h_{p, \max }^{*}$, the film deformation is restricted by the bottom substrate, and as such the drop deformation is enhanced (figure 1c). Furthermore, the deformation of the liquid surface not only converts part of the drop kinetic energy away, but it also forms a crater that limits the spreading of the drop. As $H^{*}$, and thus $H^{*}-h_{p, \max }^{*}$ decreases, the drop deformation increases due to the combination of the increased resistance from the bottom substrate and the reduced liquid film deformation.

Figure 3(b) shows the effect of We on drop spreading by comparing $\beta$ as a function of We for both the deep and shallow pool limits for S05 (other liquids also show similar behaviour). Since the impact inertia and surface tension are respectively the driving and restoring forces for the drop spreading, we observe monotonic increase in $\beta$ with their ratio, i.e. We. The scatter of the data in the shallow pool limit comes from the strong influence of the film thickness, as shown in figure 3(a). This effect, however, is not present in the deep pool limit and hence the data are significantly less scattered. The dependence of $\beta$ on We for the deep pool is observed consistently for all the liquids with a range of viscosities (figure $3 c$ ). It also shows that various liquids follow the scaling of $\beta_{D P} \sim W e^{0.066}$, demonstrating a weak effect of We on spreading in the deep pool limit. Moreover, overlap of the data from various liquids with a range of viscosities also suggests a weak effect of viscosity on the spreading factor. These results imply that the impact kinetic energy is mostly used for the deformation of the liquid film, rendering the drop deformation weaker. The spreading factors are further compared with those for drop impacts on solid surfaces in the similar We range reported in the literature (Clanet et al. 2004; Tran et al. 2012; Wildeman et al. 2016) (figure $3 c$ ). It is seen that for small $W e(<3), \beta$ from impacts on solid surfaces and deep pools follow a similar, albeit weak, dependence on We, while for moderate to high $\mathrm{We}(>3)$, impacts on solid surfaces show a sharper increase in $\beta$ with We. It is also noted that for all We ranges, $\beta$ for impacts on deep pools is significantly smaller than that on solid surfaces. Such a drastic difference is expected since drop impacts on liquid surfaces are fundamentally different from those on solid surfaces, in that the former involve impacts on a deformable surface which can adapt to the impact; while for the latter, the drop motion is restricted by the impacted rigid surface, whose properties including roughness and wettability come into play. Moreover, impacts on the liquid surfaces, especially in the deep pool limit, not only cause significant deformation of the liquid surface (figure $1 b$ ), but also induce motion in the liquid film, both of which absorb substantial impact kinetic energy, and hence limit the effective kinetic energy left for the drop to deform. The cumulative effect causes the spreading factor smaller for the impact on liquid surfaces.

\subsection{Scaling analysis}

We next perform a scaling analysis to assess the effects of $W e$ and $H^{*}$ on the spreading factor in both limits of a deep pool and a shallow pool. Considering an energy balance between before the impact and at the maximum spreading, where the kinetic energy of the drop can be neglected, we can write $(K E)_{D, 0}=(K E)_{F}+(\Delta S E)_{D}+(\Delta S E)_{F}+E_{\phi}$. Here, $(K E)_{D, 0}=(4 / 3) \pi R^{3} \rho U^{2}$ is the kinetic energy of the drop before impact; $(K E)_{F}$ is the kinetic energy of the liquid film induced by the impact, which is $(3 / 4)(K E)_{D, 0}$, as shown in previous studies (Tran et al. 2013; Tang et al. 2016, 2018); $(\Delta S E)_{D}$ and $(\Delta S E)_{F}$ are the changes in the surface energy due to changes in the drop and film shapes respectively; and $E_{\phi}$ is the energy loss due to 
viscous dissipation. For the deep pool $\left(H^{*}>h_{p, \max }^{*}\right)$, where we assume that the deformation of the liquid film takes the shape of a cylindrical well, as shown in figure $1(b)$, such that the surface energies scale as $(\Delta S E)_{F} \approx 2 \pi R_{\max , D P} h_{p, \max } \sigma$ and $(\Delta S E)_{D} \approx 4 \pi R_{\max , D P}^{2} \sigma$. The boundary layer thickness normalized by drop diameter at the time of maximum deformation, can be estimated by the momentum diffusion thickness, $L_{b} / 2 R \approx \sqrt{v t_{m}} / 2 R=\sqrt{(\pi / 4) O h\left(t_{m} / \tau_{c a p}\right)}$, where $L_{b}$ is the boundary layer thickness (Roisman 2009; Visser et al. 2015; Wildeman et al. 2016). The normalized time taken by the drop to reach the maximum deformation $\left(t_{m} / \tau_{c a p}\right)$ in the current study is less than 0.5. Since the Ohnesorge numbers $(O h=v \sqrt{\rho / \sigma R})$ for the liquids reported here are less than 0.01 , we expect the viscous dissipation to be confined to a thin boundary layer $\left(L_{b} / 2 R<0.06\right)$ and hence, it is neglected, i.e. $E_{\phi} \approx 0$. The collapse of $\beta$ for all liquids (figure $3 c$ ) also supports this assumption. Substituting these expressions in the energy balance, for the deep pool, we find

$$
\frac{W e}{12} \approx h_{p, \max }^{*} \beta_{D P}+2 \beta_{D P}^{2},
$$

where $\beta_{D P}=\left(R_{\max , D P} / R\right)$ is the spreading factor in the deep pool limit. As expected, $\beta_{D P}$ is independent of $H^{*}$ since the impact is not affected by the bottom substrate, which is consistent with the data in figure 3(a). Our previous experimental data (Tang et al. 2018) on transition between bouncing and merging states for a drop impact on a deep pool, showed that the normalized penetration depth $\left(h_{p, \max }^{*}\right)$, for a wide range of liquids, is linearly dependent on We, i.e. $h_{p, \max }^{*} \sim W e$. If we assume $\beta_{D P} \sim W e^{n}$, the scaling dependence of (4.1) can be rewritten as $W e / 12 \sim W e^{1+n}+2 W e^{2 n}$. For the right-hand side to match the linear dependence on We of the left-hand side, $n$ must be negligible. In other words, we expect $\beta$ to show a very weak dependence on $W e$ in the deep pool limit, which agrees with the exponent of 0.066 shown in figure $3(c)$.

In the shallow pool limit $\left(H^{*}<h_{p, \max }^{*}\right)$, however, the deformation of the film $\left((\Delta S E)_{F}\right)$, is restricted by the bottom substrate, and the drop spreading $\left((\Delta S E)_{D}\right)$ is enhanced, as illustrated in figure 1(c). Following figure 1(c) for an arbitrary film thickness $H$, the reduction in the deformed film surface energy with respect to a deep pool can be expressed as $\delta(\Delta S E)_{F} \approx 2 \pi\left(R_{\max , S P}\left(h_{p, \max }-H\right)\right) \sigma$, while the increase in the deformed drop surface energy with respect to deep pool can be expressed as $\delta(\Delta S E)_{D} \approx 4 \pi\left(R_{\max , S P}^{2}-R_{\max , D P}^{2}\right) \sigma$. Subsequently, comparing the deep and shallow pool limits and assuming that the reduction in the surface energy of the film leads to the increase in the surface energy of the drop, i.e. $\delta(\Delta S E)_{F}=\delta(\Delta S E)_{D}$, for the shallow pool limit we find

$$
\beta_{S P}^{2}-\beta_{D P}^{2} \approx \frac{\beta_{D P}}{2}\left(h_{p, \max }^{*}-H^{*}\right),
$$

where $\beta_{S P}=R_{\max , S P} / R$ is the drop deformation in the shallow pool limit. Note that the spreading factor for the shallow pool limit approaches that for the deep pool limit $\left(\beta_{S P} \rightarrow \beta_{D P}\right)$ as the film thickness approaches the transition boundary $\left(H^{*} \rightarrow h_{p, \max }^{*}\right)$. Substituting $\beta_{D P}$ from (4.1) into (4.2) gives

$$
\beta_{S P}^{2} \approx \frac{W e}{24}-H^{*} \beta_{D P}
$$

which leads to $\beta_{S P} \sim \sqrt{W e}$ at the limit of vanishing film thickness. To test the scaling for the shallow pool limit with the experiment, in figure $3(d)$ we plot the experimentally measured $\beta_{S P}^{2}$ as a function of $W e$ for various $H^{*}$ for all liquids tested, which shows a linear dependence. We also see that the value of $\beta_{S P}$ progressively decreases with $H^{*}$ for a given $W e$. Both of these support the scaling expressed in (4.3). 

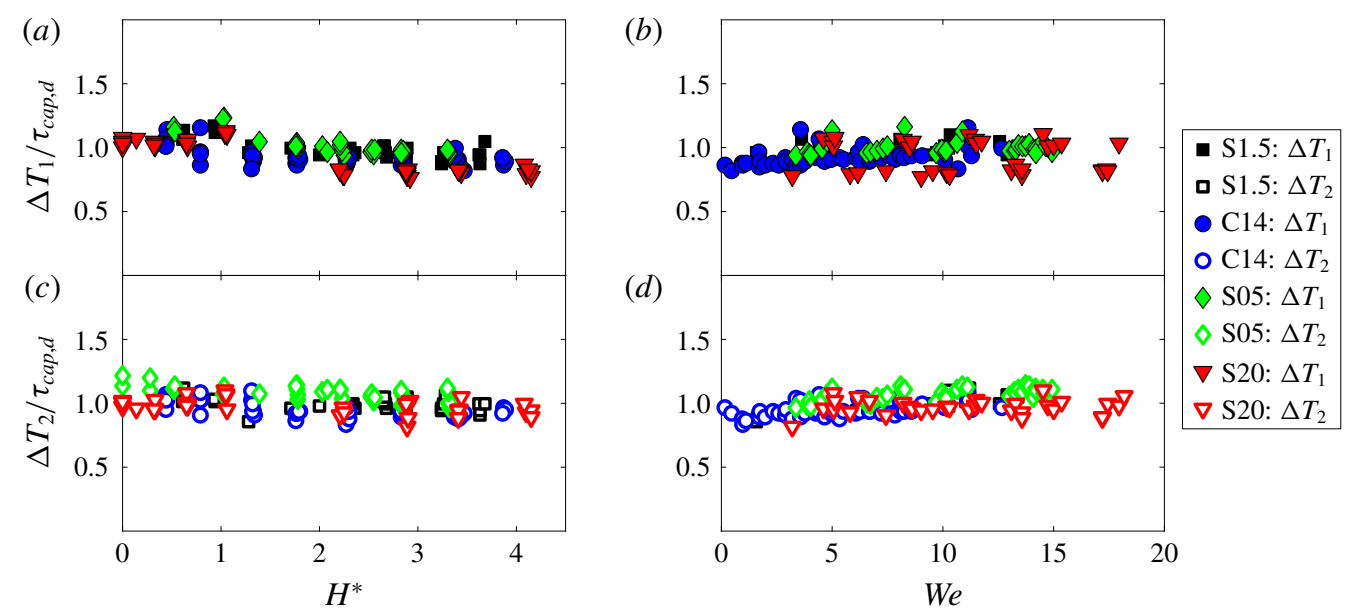

FIgURE 4. (Colour online) Time scale of the drop shape oscillation. Time period between peaks $\Delta T_{1}$, normalized by damped capillary time scale as a function of $(a) H^{*}$ and $(b) W e$. Time period between valleys $\Delta T_{2}$, normalized by damped capillary time scale $\tau_{c a p, d}$ as a function of $(c) H^{*}$ and $(d)$ We. The $\Delta T_{i}$ are defined in figure $2(c)$.

\section{Oscillation dynamics}

\subsection{Oscillation time scale}

The oscillation dynamics of the drop shape beyond the initial spreading (figure $2 c$ ) resembles a damped oscillator. To analyse this behaviour, we use the reference of a freely oscillating drop, whose oscillation time scale $\tau_{\text {cap }}$ and frequency $\omega_{\text {cap }}$ for an inviscid liquid are given by $\omega_{c a p}=2 \pi / \tau_{c a p}=\sqrt{(8 \sigma) /\left(\rho R^{3}\right)}$ (Lifshitz \& Landau 1959). For a real fluid, however, the oscillation is affected by viscous dissipation resulting in a damped oscillation frequency: $\omega_{c a p, d}=\omega_{c a p} \sqrt{\left(1-\varepsilon^{2}\right)}=2 \pi / \tau_{c a p, d}$. Here, $\tau_{c a p, d}$ is the time scale for the damped oscillation and $\varepsilon$ is the damping ratio: $\varepsilon=\eta / \omega_{\text {cap }}$, where $\eta \approx 8 v / R^{2}$ is the damping coefficient. The damping ratio can thus be further related to the Ohnesorge number: $\varepsilon=2 \sqrt{2} \mathrm{Oh}$.

Now, we compare the time periods between consecutive peaks and valleys of the observed damped oscillation with the theoretical values. In figure $4(a, c)$, the time periods normalized by the damped capillary time scale $\tau_{\text {cap }, d}$ are plotted as a function of $H^{*}$, where the closed and open symbols represent $\Delta T_{1}$ and $\Delta T_{2}$ respectively (defined in figure $2 c$ ) and symbols and colours represent different liquids. We observe no significant variations for different $H^{*}$, which indicates that neither the deformation of the liquid surface nor the resistance from the solid substrate affects the oscillation frequency. Furthermore, the normalized periods not only are independent of We (figure $4 b, d$ ), they also assume a mean value around unity. This signifies that the frequencies of the post-impact oscillations are primarily controlled by capillarity and viscosity, and are not affected by the impact inertia.

\subsection{Viscous decay}

While the kinetic and surface energies are periodically interchanged during the oscillation, part of the total energy is continuously dissipated through viscous loss and, as such, the maximum radius progressively decays after each cycle. This decay can be quantified by the ratio between the second $\left(R_{2}\right)$ and first, which is 

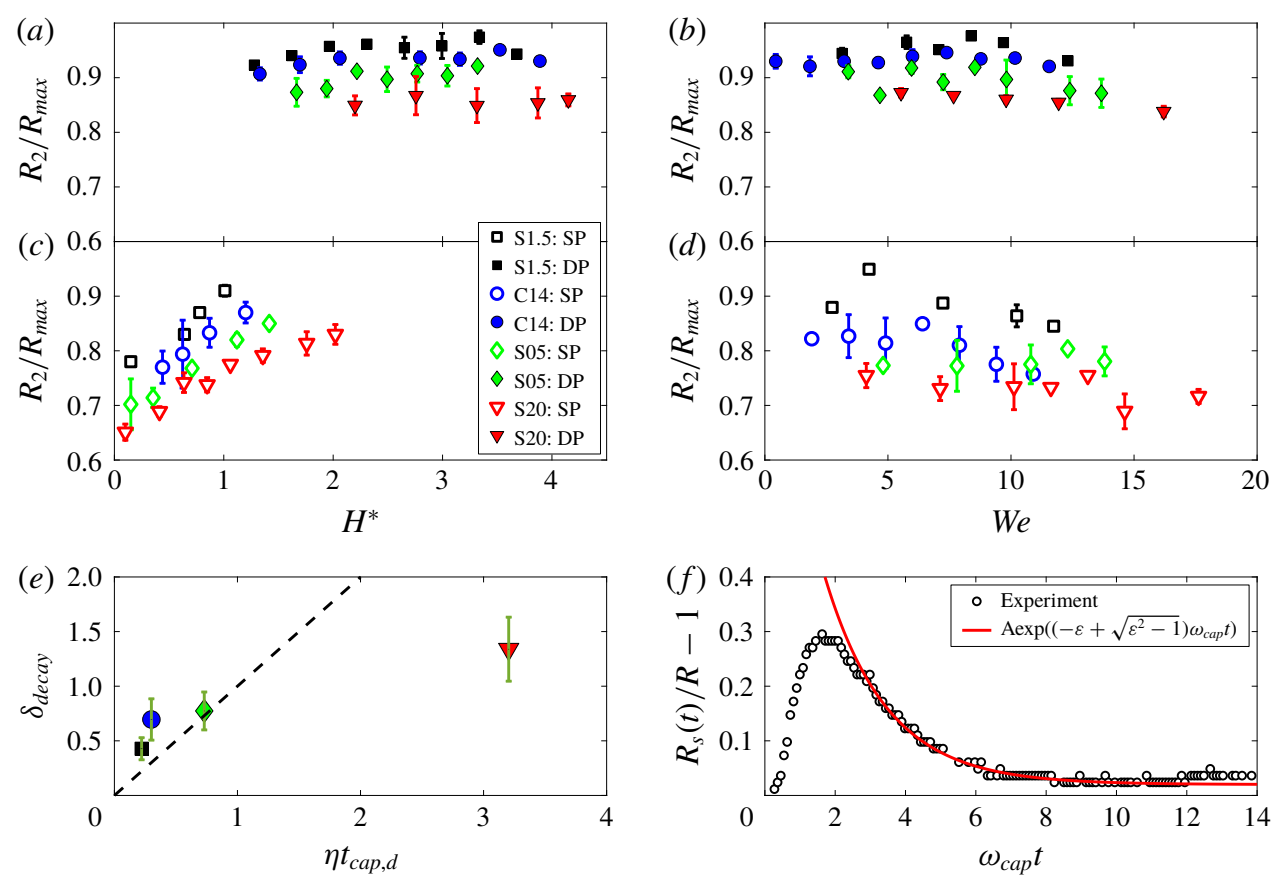

Figure 5. (Colour online) Viscous decay of drop oscillation. ( $a-d)$ Ratio of second peak to first peak in observed oscillations as a function of $H^{*}$, for $(a)$ deep pool and $(c)$ shallow pool. Ratio of second peak to first peak in observed oscillations as a function of $W e$, for $(b)$ deep pool and $(d)$ shallow pool. (e) Logarithmic decrements as a function of $\eta t_{c a p, d}$. Error bar shows the scatter in the data. The line shows the linear function for a system with linear damping. $(f)$ Overdamping behaviour of S100. Line: theoretical prediction based on equation: $R(t) / R_{s}-1 \approx A \exp \left(\left(-\varepsilon+\sqrt{\varepsilon^{2}-1}\right) \omega_{\text {cap }} t\right)$ for $A=1$. Circle: experimental data.

the maximum radius $\left(R_{\max }\right.$, defined in figure $2 c$ ), which is plotted against $H^{*}$ in figures $5(a)$ and $5(c)$ for all the liquids. We observe a strong dependence of the viscous decay on the film thickness. Specifically, in the shallow pool limit, the decay becomes weaker with increasing film thickness (figure $5 a$ ), until it asymptotically attains an almost constant value in the deep pool limit (figure $5 c$ ). For small film thicknesses, the drop spreads over the bottom substrate, which leads to a strong flow gradient inside the drop, and hence stronger viscous dissipation and damping in the oscillation. As the film thickness increases, the drop deformation, thus dissipation, weakens. At the deep pool limit, the effect of the film thickness vanishes, and the dissipation is only induced by the internal motion of the weakly deformed drop which is independent of the film thickness, resulting in an almost constant decay with respect to $H^{*}$. Figure $5(b)$ shows that the viscous decay for the deep pool limit is mostly independent of We except for the high-viscosity liquids such as S20, for which the decay slightly increases with $W e$. The analysis of nonlinear oscillation of a freely oscillating viscous drop (Basaran 1992) shows that viscous damping increases with both the viscosity $(O h)$ and the initial deformation $(\beta)$, where the latter is larger for higher $W e$, as shown in the previous section. In the shallow pool limit, since the initial deformation caused by the spreading $(\beta)$ is even higher, the positive dependence of the viscous decay on $W e$ is more prominent (figure $5 d$ ). To quantify the viscous decay, we examined the logarithm of the ratio of neighbouring 
peaks, namely the logarithmic decrement, defined as $\delta_{\text {decay }}=\ln \left[\left(R_{\max }-R\right) /\left(R_{2}-R\right)\right]$. For a linearly damped oscillation, the logarithmic decrement can be expressed as $\delta_{\text {decay }} \sim \eta \tau_{\text {cap }, d}$. In figure 5(e), we compared the measured $\delta_{\text {decay }}$ with $\eta \tau_{c a p, d}$ for the deep pool limit, which shows qualitative agreement, i.e. the decay increases with increasing viscosity. The expected linear dependence is not observed because of the nonlinear viscous damping. To accurately predict the nonlinear viscous damping behaviour, a detailed solution of the flow field in the drop is required and cannot be obtained by a simple model. The large scatter in $\delta_{\text {decay }}$ for a single liquid comes from the weak dependence of decay on We.

Theoretically, an oscillating system loses its periodicity and displays aperiodic behaviour if the damping is too large, triggering the over-damping phenomenon. Following the linear damping model discussed above, over-damping happens when the damping ratio is larger than unity, i.e. $\varepsilon>1$, or $O h>0.35$, for a damped oscillating drop. In our experiments, the viscosity of S100 is significantly larger than other liquids and satisfies the over-damping condition $(O h=0.88>0.35)$. The shape oscillations after the initial spreading stage are indeed significantly damped for S100 (figure $5 f$ ) with no periodic behaviour. Using the theoretical solution for the over-damped system $R(t) / R_{s}-1 \approx A \exp \left(\left(-\varepsilon+\sqrt{\varepsilon^{2}-1}\right) \omega_{\text {cap }} t\right)$, the behaviour of the drop radius can be well predicted, as shown in figure $5(f)$.

\section{Conclusion}

In summary, we have investigated the spreading and oscillation dynamics when drops of different We and viscosities bounce upon impacting liquid surfaces of various film thicknesses. The spreading dynamics, characterized by the spreading factor, has negligible dependence on the film thickness in the deep pool regime as the drop kinetic energy is primarily converted to surface energy of the significantly deformed film surface. On the other hand, in the shallow pool regime, the spreading factor decreases with $H^{*}$ and increases with $W e$, since the film deformation is inhibited and drop deformation is promoted by the bottom substrate. The viscous effect has little influence on the spreading dynamics. The oscillation time scale follows that of a freely oscillating drop with no dependence on $W e$ and $H^{*}$. The viscous decay of the oscillation is not affected by $W e$ and $H^{*}$ in the deep pool regime. However, in the shallow pool regime, the decay decreases with $H^{*}$ and increases with $W e$, owing to enhanced viscous loss due to the higher degree of drop deformation. Over-damping behaviour is observed and predicted for S100 with a viscosity beyond the critical value.

\section{Acknowledgements}

The work performed at Princeton University was supported by the Army Research Office (grant no. W911NF1610449) under the technical monitoring of $\mathrm{Dr}$ R. A. Anthenien.

\section{REFERENCES}

Basaran, O. A. 1992 Nonlinear oscillations of viscous liquid drops. J. Fluid Mech. 241, 169-198. Basaran, O. A., SCOTT, T. C. \& Byers, C. H. 1989 Drop oscillations in liquid-liquid systems. AIChE J. 35 (8), 1263-1270.

Becker, E., Hiller, W. J. \& Kowalewski, T. A. 1991 Experimental and theoretical investigation of large-amplitude oscillations of liquid droplets. J. Fluid Mech. 231, 189-210. 
Becker, E., Hiller, W. J. \& Kowalewski, T. A. 1994 Nonlinear dynamics of viscous droplets. J. Fluid Mech. 258, 191-216.

Clanet, C., Béguin, C., Richard, D. \& Quéré, D. 2004 Maximal deformation of an impacting drop. J. Fluid Mech. 517, 199-208.

Cossali, G. E., Coghe, A. \& Marengo, M. 1997 The impact of a single drop on a wetted solid surface. Exp. Fluids 22 (6), 463-472.

VAn DAM, D. B. \& LE ClerC, C. 2004 Experimental study of the impact of an ink-jet printed droplet on a solid substrate. Phys. Fluids 16 (9), 3403-3414.

Davidson, M. R. 2002 Spreading of an inviscid drop impacting on a liquid film. Chem. Engng Sci. 57 (17), 3639-3647.

Eggers, J., Fontelos, M. A., Josserand, C. \& Zaleski, S. 2010 Drop dynamics after impact on a solid wall: theory and simulations. Phys. Fluids 22 (6), 062101.

GAO, X. \& LI, R. 2015 Impact of a single drop on a flowing liquid film. Phys. Rev. E 92, 053005.

Geppert, A., Terzis, A., Lamanna, G., Marengo, M. \& Weigand, B. 2017 A benchmark study for the crown-type splashing dynamics of one-and two-component droplet wall-film interactions. Exp. Fluids 58 (12), 172.

Josserand, C. \& Thoroddsen, S. T. 2016 Drop impact on a solid surface. Annu. Rev. Fluid Mech. 48 (1), 365-391.

Josserand, C. \& Zaleski, S. 2003 Droplet splashing on a thin liquid film. Phys. Fluids 15 (6), $1650-1657$.

Lagubeau, G., Fontelos, M. A., Josserand, C., Maurel, A., Pagneux, V. \& Petitjeans, P. 2010 Flower patterns in drop impact on thin liquid films. Phys. Rev. Lett. 105, 184503.

Lifshitz, E. M. \& LandaU, L. D. 1959 Fluid Mechanics, Course of Theoretical Physics, vol. 6. (2nd ed.). Butterworth-Heinemann.

Lundgren, T. S. \& MANSOUR, N. N. 1988 Oscillations of drops in zero gravity with weak viscous effects. J. Fluid Mech. 194, 479-510.

Marcotte, F., Michon, G.-J., SÉon, T. \& Josserand, C. 2019 Ejecta, corolla, and splashes from drop impacts on viscous fluids. Phys. Rev. Lett. 122, 014501.

MashayeK, F. \& Ashgriz, N. 1998 Nonlinear oscillations of drops with internal circulation. Phys. Fluids 10 (5), 1071-1082.

Miller, C. A. \& SCRiven, L. E. 1968 The oscillations of a fluid droplet immersed in another fluid. J. Fluid Mech. 32 (3), 417-435.

PAN, K. \& LAW, C. K. 2007 Dynamics of droplet-film collision. J. Fluid Mech. 587, 1-22.

Prosperetti, A. 1980 Free oscillations of drops and bubbles: the initial-value problem. J. Fluid Mech. 100 (2), 333-347.

REID, W. H. 1960 The oscillations of a viscous liquid drop. Q. J. Math. 18 (1), 86-89.

Rieber, M. \& Frohn, A. 1999 A numerical study on the mechanism of splashing. Intl J. Heat Fluid Flow 20 (5), 455-461.

Rioboo, R., Bauthier, C., Conti, J., Voue, M. \& De Coninck, J. 2003 Experimental investigation of splash and crown formation during single drop impact on wetted surfaces. Exp. Fluids 35 (6), 648-652.

RoIsman, I. V. 2009 Inertia dominated drop collisions. II. An analytical solution of the Navier-Stokes equations for a spreading viscous film. Phys. Fluids 21 (5), 052104.

Roisman, I. V., van Hinsberg, N. P. \& Tropea, C. 2008 Propagation of a kinematic instability in a liquid layer: capillary and gravity effects. Phys. Rev. E 77, 046305.

Roisman, I. V., Rioboo, R. \& Tropea, C. 2002 Normal impact of a liquid drop on a dry surface: model for spreading and receding. Proc. R. Soc. Lond. A 458 (2022), 1411-1430.

Saha, A., WeI, Y., TAng, X. \& LAW, C. K. 2019 Kinematics of vortex ring generated by a drop upon impacting a liquid pool. J. Fluid Mech. 875, 842-853.

Schiaffino, S. \& Sonin, A. A. 1997 Molten droplet deposition and solidification at low Weber numbers. Phys. Fluids 9 (11), 3172-3187.

SoN, Y. \& KIM, C. 2009 Spreading of inkjet droplet of non-Newtonian fluid on solid surface with controlled contact angle at low Weber and Reynolds numbers. J. Non-Newtonian Fluid Mech. 162 (1), 78-87. 
TANG, X., SAHA, A., LAw, C. K. \& Sun, C. 2016 Nonmonotonic response of drop impacting on liquid film: mechanism and scaling. Soft Matt. 12 (20), 4521-4529.

TANG, X., SAHA, A., LAW, C. K. \& SUn, C. 2018 Bouncing-to-merging transition in drop impact on liquid film: role of liquid viscosity. Langmuir 34 (8), 2654-2662.

TAng, X., Saha, A., LAw, C. K. \& Sun, C. 2019 Bouncing drop on liquid film: dynamics of interfacial gas layer. Phys. Fluids 31 (1), 013304.

Tran, A. T. T., Hyland, M. M., Shinoda, K. \& Sampath, S. 2011 Influence of substrate surface conditions on the deposition and spreading of molten droplets. Thin Solid Films $\mathbf{5 1 9}$ (8), 2445-2456.

Tran, T., De Maleprade, H., Sun, C. \& Lohse, D. 2013 Air entrainment during impact of droplets on liquid surfaces. J. Fluid Mech. 726, R3.

Tran, T., Staat, H. J. J., Prosperetti, A., Sun, C. \& Lohse, D. 2012 Drop impact on superheated surfaces. Phys. Rev. Lett. 108 (3), 036101.

TRINH, E. \& WANG, T. G. 1982 Large-amplitude free and driven drop-shape oscillations: experimental observations. J. Fluid Mech. 122, 315-338.

Trinh, E. H., Thiessen, D. B. \& Holt, R. G. 1998 Driven and freely decaying nonlinear shape oscillations of drops and bubbles immersed in a liquid: experimental results. J. Fluid Mech. 364, 253-272.

Tsamopoulos, J. A. \& Brown, R. A. 1983 Nonlinear oscillations of inviscid drops and bubbles. J. Fluid Mech. 127, 519-537.

Vadillo, D. C., Soucemarianadin, A., Delattre, C. \& Roux, D. C. D. 2009 Dynamic contact angle effects onto the maximum drop impact spreading on solid surfaces. Phys. Fluids 21 (12), 122002.

Visser, C. W., Frommhold, P. E., Wildeman, S., Mettin, R., Lohse, D. \& Sun, C. 2015 Dynamics of high-speed micro-drop impact: numerical simulations and experiments at frameto-frame times below 100 ns. Soft Matt. 11 (9), 1708-1722.

Wildeman, S., Visser, C. W., Sun, C. \& Lohse, D. 2016 On the spreading of impacting drops. J. Fluid Mech. 805, 636-655.

YARIN, A. L. 2006 Drop impact dynamics: splashing, spreading, receding, bouncing ... Annu. Rev. Fluid Mech. 38, 159-192. 